\title{
POPOLO DELLA LIBERTÀ: AUGE Y CAÍDA DE UN PARTIDO CONSERVADOR'
}

\author{
Popolo della Libertà: the rise and fall of a \\ conservative party
}

\author{
JORGE DEL PALACIO MARTÍN \\ Universidad Rey Juan Carlos \\ jorge.delpalacio@urjc.es
}

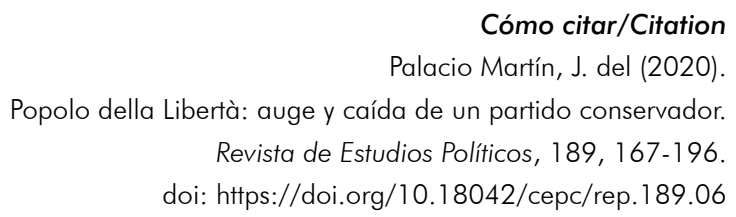

Resumen

El presente artículo analiza la dimensión ideológica del partido de Silvio Berlusconi el Popolo della Libertà (2008-2013). La atención a la naturaleza ideológica del PdL se justifica porque su fracaso como partido conservador resulta un episodio crucial para entender la metamorfosis de la derecha italiana en el marco de la transformación del sistema de partidos que se produce en la década 2008-2018. El análisis concluye que la superación de la competición bipolar que caracterizó la Segunda República ha contribuido a reducir la capacidad de Berlusconi para movilizar al electorado hostil a la izquierda en clave anticomunista.

\section{Palabras clave}

Ideología; liberalismo; conservadurismo; anticomunismo; populismo; Italia.

1 Este trabajo se ha beneficiado de una estancia en el International Center on Democracy and Democratization de la Universidad LUISS-Guido Carli de Roma. Agradezco a su director, Giovanni Orsina, como al profesor Luis Arranz, los comentarios y sugerencias a los primeros borradores de este artículo. 


\section{Abstract}

The following article analyses the ideological dimension of Silvio Berlusconi's political party Popolo della Libertà (2008-2013). The focus on the ideological nature of the PdL is justified because its failure as a conservative party is a crucial factor, along with the transformation of the political party system in Italy, in understanding the metamorphosis of the Italian right between 2008 and 2018. The analysis concludes that the demise of the two-party system that characterized the Second Republic has contributed to reduce Berlusconi's traditional ability to mobilise the anti-left wing electorate through an anticommunist discourse.

\section{Keywords}

Ideology; liberalism; conservatism; anticommunism; populism; Italy. 


\section{SUMARIO}

I. INTRODUCCIÓN. II. LA POLÍTICA DE LA SEGUNDA REPÚBLICA: LA COMPETICIÓN BIPOLAR Y LA FUNDACIÓN DEL POPOLO DELLA LIBERTÀ: 1. La lógica de competición bipolar y el nacimiento del PD y el PdL. 2. "Politica di Piazza»: la oposición al Gobierno Prodi. III. CARATERÍSTICAS IDEOLÓGICAS DEL PdL: 1. El diseño programático de un partido conservador. 2. La continuidad del discurso anticomunista. IV. HACIA LA DISOLUCIÓN DEL POPOLO DELLA LIBERTÀ: 1. La crisis interna un proyecto conservador. 2. Cambio de sistema y metamorfosis de la derecha italiana. V. CONCLUSIONES. BibLIOgRAFía.

\section{INTRODUCCIÓN}

El objetivo de este artículo es analizar la trayectoria del Popolo della Libertà en el marco de la transformación del sistema político italiano que se produce en la década 2008-2018 atendiendo, sobre todo, a su dimensión ideológica. El análisis del caso del PdL se justifica porque su auge y caída resulta un pasaje crucial para entender la metamorfosis de la derecha italiana a partir de las elecciones de 2013, últimas a las que concurrió el PdL. Si en las elecciones de 2008 el PdL consiguió una victoria reseñable al obtener el $37,38 \%$ de los votos - el mejor resultado logrado por un partido liderado por Berlusconi desde 1994-, en las de 2018 Forza Italia vio disminuido su consenso al 14\%, perdiendo, por primera vez en su historia, la condición de partido hegemónico de la derecha italiana a favor de la nueva Lega liderada por Matteo Salvini. En este sentido, la atención a la breve y conflictiva historia del Popolo della Libertà, como el complicado retorno de Forza Italia tras su refundación, se justifica porque refleja con bastante precisión los problemas de la derecha liderada por Silvio Berlusconi para adaptarse a los cambios registrados en la política italiana en el periodo 2008-2018.

A la hora de analizar la trayectoria del Popolo della Libertà se prestará especial atención a la dimensión ideológica del partido. Para ello se trabajará con programas de partido, discursos políticos, comentarios de prensa e intervenciones públicas de los protagonistas e ideólogos del partido. No se trata, como parece lógico, de tomar la ideología como la única o principal variable explicativa de la vida del partido, sino de atender a su importancia como 
instrumento de adaptación a un entorno competitivo en relación con otros factores. Según Angelo Panebianco en su obra clásica Modelos de partido, la ideología sirve a los partidos al propósito de fortalecer ad intra la identidad colectiva de la organización. A la vez, contribuye a configurar ad extra un territorio de caza sobre el que el partido está llamado a desarrollar una actividad de control y dominio. Ambas dimensiones, como se tratará de poner de manifiesto con el examen de la naturaleza ideológica del PdL, mantienen una relación dinámica y orgánica (Panebianco, 2009: 46-47).

El propósito de esta contribución es mostrar que el fracaso del Popolo della Libertà encuentra un factor explicativo en la incapacidad de la derecha liderada por Berlusconi para adaptar su discurso y configuración ideológica a la superación de la competición bipolar de la Segunda República. En este sentido, la principal tesis que defenderá este artículo es que la progresiva afirmación de un modelo de competición tripolar a partir de 2011, con una nueva oferta política anti-establishment que supera el eje izquierda-derecha, ha contribuido a menoscabar el potencial del discurso de Berlusconi para agrupar y movilizar al electorado hostil a la izquierda en clave anticomunista.

\section{LA POLÍTICA DE LA SEGUNDA REPÚBLICA: LA COMPETICIÓN BIPOLAR Y LA FUNDACIÓN DEL POPOLO DELLA LIBERTÀ}

El nacimiento oficial del Popolo della Libertà (PdL) como partido, fruto de la fusión de Forza Italia (FI) y Alleanza Nazionale (AN), tuvo lugar en el Primer Congreso Nacional del PdL, celebrado en Roma el 27 de marzo de 2009. Sin embargo, tal y como Silvio Berlusconi se encargó de subrayar en el discurso de apertura del congreso, a la altura de 2009 el PdL era «un movimiento que en realidad ya había nacido, ya había crecido, ya era fuerte y ya era ganador» (Berlusconi, 2013: 139). Al magnate milanés no le faltaba razón. El PdL, puesto en circulación como hipótesis a la altura de 2006, en 2008 había servido a Berlusconi como lista electoral, aún sin ser constituido como partido, para ganar las elecciones italianas de 2008 y hacer alcalde de Roma a Gianni Alemanno, político de AN.

A la hora de entender las razones que llevan a la fundación del PdL como partido con vocación de agrupar el voto de la derecha italiana en una sola organización, pueden señalarse dos. Primero, el proceso de agregación de partidos que incentivó la lógica de competición bipolar de las nuevas instituciones de la Segunda República. Segundo, la naturaleza de los conflictos en el seno de la coalición de la Casa della Libertà y la dinámica de oposición al segundo Gobierno de Prodi entre 2006 y 2008. 


\section{LA COMPETICIÓN BIPOLAR Y EL NACIMIENTO DEL PD Y EL PDL}

En el ámbito de las ciencias sociales el periodo de la historia de Italia que va de 1948 a 1992 suele conocerse como Primera República, mientras que la denominación Segunda República se reserva para la etapa política que arranca con las elecciones de 1994, aunque formalmente no se haya registrado ninguna cesura en la vigencia de la Constitución italiana de 1948. La idea de transición se justifica, sin embargo, en atención a la mutación radical del sistema de partidos que se produce en el periodo 1992-1994 (Grilli di Cortona, 2007). En esta periodización, 1993 marca el año en el que se celebró el referéndum que llevó a la abolición de la ley electoral proporcional utilizada desde el final de la Segunda Guerra Mundial y a su sustitución por una ley de sentido mayoritario. De otra forma, la referencia al periodo 1992-1994 seńala el tiempo en el que los principales partidos que estructuraron el sistema de partidos nacido en la posguerra desaparecieron en el curso del proceso judicial Mani pulite, también conocido como Tangentopoli, en referencia a los casos de financiación ilegal de organizaciones políticas, dejando espacio a los nuevos partidos que protagonizarán la política italiana de la Segunda República (Cota y Verzichelli, 2007: 38-39).

A pesar de que la ley electoral de 1993 volvió a ser cambiada en 2005 por una nueva ley proporcional con premio a la mayoría, las elecciones de la Segunda República — 1994, 1996, 2001, 2006 y 2008 — se han caracterizado por dar forma a una dinámica de competición bipolar que posibilitó la alternancia en el Gobierno. Una alternancia que había resultado imposible en el marco del sistema de partidos de la Primera República, debido, principalmente, a la conventio ad excludendum frente al PCI, el partido comunista más poderoso de Occidente en un mundo estructurado en torno a la fractura ideológica bipolar de la Guerra fría. En este sentido, el sistema de partidos de la Segunda República, que se afirma a partir de las elecciones de 1994, es definido como bipolar en atención, al menos, a dos criterios. El primero, la existencia de dos polos diferenciados y diferenciables que concentran la mayoría de los votos; el segundo, la posibilidad real de que cualquiera de los polos consiga una mayoría suficiente para formar Gobierno (Bartolini et al., 2004: 2).

Pasquino y Valbruzzi introducen una clave de lectura necesaria a la hora de profundizar en la comprensión de la naturaleza del bipolarismo italiano y medir su rendimiento. Ambos autores sostienen que, si bien el sistema de partidos de la Segunda República puede considerarse bipolar en relación a su mecánica, debe ser tomado como multipolar en relación a su forma. Lo que ponen de manifiesto es que el bipolarismo italiano se consolidó, paradójicamente, de la mano de una dinámica creciente de fragmentación de partidos. 
Según sus propias palabras, el particular bipolarismo italiano "ha terminado creando un caso anómalo de bipolarismo extremo donde un número considerable de partidos ha sido constreñido y encapsulado por dos coaliciones de gobierno que compiten entre sí. El tipo de bipolarismo que arraigó en Italia supuso que los gobiernos estaban condenados a la inestabilidad debido a la existencia de demasiadas diferencias programáticas entre los miembros de las coaliciones» (Pasquino y Valbruzzi, 2015: 443).

Precisamente, la capacidad de los pequeños partidos para desestabilizar la vida de las coaliciones y los Gobiernos se convirtió en la tónica dominante de la vida política de la Segunda República. Casos como la caída del primer Gobierno Berlusconi tras perder el apoyo parlamentario de la Lega Nord (1994-1995); la disolución del primer Gobierno Prodi como consecuencia de la pérdida del apoyo de Rifondazione Comunista (1996-1998), o el final del segundo Gobierno Prodi (2006-2008) tras perder el apoyo del pequeño partido democristiano UDEUR de Clemente Mastella, ejemplifican la realidad de un bipolarismo frágil e inestable condicionado en sumo grado por socios de coalición menores.

Esta circunstancia fue dando cuerpo en Italia a un debate sobre la necesidad de racionalizar y optimizar la competición política neutralizando el poder de bloqueo que el sistema bipolar había puesto a disposición de los pequeños partidos. Precisamente, la creación del Partito Democratico en 2007, como la fundación del Popolo della Libertà en 2009, respondían al proyecto de simplificar el sistema político a través de la conversión de las coaliciones en grandes partidos con vocación mayoritaria, siguiendo la expresión duvergeriana. El primero encontraba su origen en la fusión de los exdemocristianos de La Margherita y los excomunistas Democratici di Sinistra; el segundo en la fusión de Forza Italia con Alleanza Nazionale, el partido postfascista liderado por Gianfranco Fini.

El lema «corriamo da soli», puesto en circulación por Walter Veltroni, primer secretario del PD y alcalde de Roma entre 2001 y 2008, ilustraba la vocación mayoritaria del partido llamado a representar la mayoría del centro-izquierda italiano. Sobre todo de emanciparse de la tradicional dependencia de Rifondazione Comunista para poder llevar el partido hacia el centro ${ }^{2}$. Su objetivo, como vendría a decir Veltroni en el congreso fundacional del PD, era crear «el partido del nuevo milenio y de la libertad, que desafiará a los conservadurismos de derecha e izquierda $»^{3}$. La fundación del PD supuso

\footnotetext{
M. Salvati «La battaglia del centro», Corriere della Sera, 26-03-2008.

3 "Veltroni: Fare un'Italia nuova», Corriere della Sera, 27-01-2007. En agosto del mismo año Veltroni publicó un libro-manifiesto llamado significativamente La nuova stagione.
} 
un estímulo para Silvio Berlusconi, que desde su salida del Gobierno en 2006 había acariciado la idea de superar las acentuadas desavenencias entre los partidos de su coalición a través de la fundación de un nuevo sujeto político unitario para la derecha italiana, un proyecto que puede verse delineado en su libro Verso il partito della libertà, publicado a comienzos de 2006, en el que la colección de discursos que lo componen permite seguir la pista del proyecto de fundación de un «nuovo partito»"

\section{2. «POLITICA DI PIAZZA»: LA OPOSICIÓN AL GOBIERNO PRODI}

El segundo Gobierno Berlusconi 2001-2005 resultó ser el más longevo de la historia política de la Italia republicana al alcanzar los 1412 días de duración ${ }^{5}$. Sin embargo, ese éxito no se tradujo en un proceso de mayor integración de los partidos que formaban la coalición Casa della Libertà. Al contrario, la vida de este Ejecutivo sirvió para ahondar las diferencias entre las cuatro almas de la derecha italiana - la federalista, la postfascista, la democristiana y la liberal, representada a su manera por el berlusconismo-, dando lugar a tensiones constantes en la coalición de Gobierno que solamente el liderazgo de Berlusconi consiguió equilibrar y neutralizar, convirtiendo a FI en el centro de gravedad de las coaliciones de centro-derecha (Diamanti y Lello, 2005: 30-31).

La XIV legislatura italiana estuvo a atravesada por debates políticos que pusieron de manifiesto la distancia que mediaba entre las culturas políticas a las que daban expresión Forza Italia, Lega Nord, Alleanza Nazionale y Unione di Centro. Unas diferencias que prefiguraban las fracturas ideológicas que separarían a la derecha italiana a partir de 2013. El europeísmo de Casini contrastaba con la ambigüedad de Umberto Bossi y Gianfranco Fini, si bien de signo opuesto, ante la Unión Europea. La cuestión del federalismo enfrentó a la Lega Nord con Alleanza Nazionale como en los ańos noventa. La Ley Bossi-Fini de inmigración (30-07-2002, n. ${ }^{\circ}$ 189) encontró la oposición de Casini, quien actuó como correa de transmisión de la opinión del mundo católico y el Vaticano sobre la materia ${ }^{6}$, mientras que el entusiasmo filo

Contro tutti y conservatorismi (Rizzoli, 2007).

4 El documento "Manifesto dei valori», del 8 de noviembre de 2005, aprobado y suscrito por los miembros de la Asamblea Constituyente del partido unitario de la CdL, delinea la línea programática de un nuovo partito (Berlusconi, 2006: 153-183).

5 En la XIV legislatura también se registra el Gobierno Berlusoni III, constituido el 23 de abril de 2005 y finalizado el 16 de mayo de 2006.

6 En una encuesta realizada por LaPolis de cara a las elecciones europeas de 2004, el $32,4 \%$ del electorado de UdC respondió positivamente a la pregunta «¿Los inmigrantes 
americano de la política exterior de Berlusconi, con el corolario del respaldo a intervención en Irak, no siempre encontró eco en las filas de sus socios de coalición (Colarizi y Gervasoni, 2012: 126 y 154-159).

A medida que las desavenencias entre sus socios de Gobierno se iban afirmando sobre todas las materias importantes en torno a las cuales había girado la legislatura, Berlusconi se enfrentó a las elecciones de 2006 poniendo sordina a los conflictos internos de la coalición CdL recurriendo al mínimo denominador común del centro derecha italiano: el anticomunismo. De este modo, Berlusconi afrontó sus últimos meses en el Palazzo Chigi haciendo campaña electoral contra la izquierda desde el Gobierno: «Si la izquierda llega al Gobierno, este será el resultado: miseria, terror y muerte. Así como ocurre allí donde gobierna el comunismo. No es el Estado liberal que nosotros queremos» ${ }^{7}$. Un movimiento que repetía la estrategia de activación del consenso anticomunista desde el poder al que Berlusconi apeló a finales de 1994, en los últimos días de su primer Gobierno: «No permitiremos nunca que entreguen Italia a los comunistas y D'Alema» ${ }^{8}$.

Sin embargo, la derrota electoral de la Casa della Libertà frente a la coalición de centro izquierda del L'Ulivo liderada por Romano Prodi en las elecciones de 2006 sirvió para fortalecer el diagnóstico que Silvio Berlusconi había madurado a lo largo de una década sobre el centro derecha italiano y su rendimiento político: si Forza Italia no había sido capaz de llevar a buen puerto las reformas institucionales necesarias para realizar la revolución liberal, ello se debía, en buena medida, a que sus aliados de Gobierno habían obstaculizado, no pocas veces, su acción de gobierno (Cicchito, 2019: 126-127).

A partir de este diagnóstico, Berlusconi desarrolló una estrategia de oposición total al nuevo Gobierno Prodi. Los días 17 y 18 de noviembre de 2007 el magnate milanés organizó jornadas de protesta contra el Gobierno, en las que recogió ocho millones de firmas en toda Italia para pedir su dimisión. La estrategia de oposición se apoyó en un discurso populista que hacía de Berlusconi el «uomo della providenza» llamado a liberar al pueblo italiano de un Gobierno de izquierda ${ }^{9}$. Como diría en el programa Otto e mezzo algunos días después de la recogida de firmas:

son un peligro para la seguridad pública?», en comparación con el 46,3\% de AN, el 50,1 \% de FI y el 56,9\% de LN (Diamanti y Lello: 2005: 28)

7 «Berlusconi: con la sinistra miseria, morte e terrore», la Repubblica, 16-01-2005.

8 «Non lascerò il paese ai comunisti», Corriere della Sera, 27-12-1994.

9 Para una interpretación populista del berlusconismo, véanse Orsina (2013), Tarchi (2015), Biorcio (2015) y Del Palacio (2017). 
Ha ocurrido algo que no había pasado en la historia de la República y yo no podía no tomar nota de este momento que la historia ha puesto ante nosotros. Ocho millones de italianos en dos días y medio han dado vida a una movilización extraordinaria para decir basta a este Gobierno y para pedir elecciones [...]. La gente me ha dado una responsabilidad muy grande y no me corresponder el mérito, son millones de italianos los que me piden, los que nos piden, cambiar Italia (Moroni, 2008: 54).

Esta imagen de un pueblo auténtico y genuino que se moviliza y actúa al margen de los políticos será explotada por Berlusconi para crear la imagen del PdL como un partido que surge de una movilización política espontánea con origen en la sociedad. La impronta de la recogida de firmas contra Prodi y su interpretación como referéndum aparece en un lugar preferente del discurso que Berlusconi ofreció en el Primer Congreso Nacional del PdL, en 2009:

Fue, aquella del 17 y 18 de noviembre, una consulta que vio fluir y registrarse espontáneamente en nuestras urnas a millones de italianos. Un pueblo auténtico, genuino, extraño a los ritos de Palacio, porque no había candidatos prefabricados que aprobar ni aparatos de partido y nomenclaturas que confirmar, nada, en suma, que pueda parecerse a los rituales de las distintas primarias de la izquierda a las que hemos asistido. Había exclusivamente una libertad de elección que realizar. Y la elección nos ha dado, por una grandísima mayoría, la siguiente indicación: tenemos que ser un pueblo, antes incluso que un partido: el Popolo della Libertà (Berlusconi, 2013: 141).

Sin embargo, resulta necesario subrayar que la movilización del electorado de la derecha orquestada por Berlusconi no solo pretendía teatralizar al pueblo, espontáneamente organizado, en acción contra el Gobierno. También era un movimiento estratégico dirigido a reafirmar su propia legitimidad como líder super partes de la derecha italiana, que respondía a las críticas y movimientos estratégicos de Bossi, Fini y Casini tras la derrota electoral de 2006, mostrando el afecto que le profesaba el pueblo. De forma aparentemente improvisada, sin contar con ninguno de los líderes de la coalición CdL, el 17 de noviembre de 2007 Berlusconi aprovechó su presencia en la recogida de firmas contra Prodi que se celebró en la plaza San Babila de Milán para anunciar su intención de disolver Forza Italia y fundar un nuevo partido. "Aquí, en medio del pueblo, nace el Popolo della Libertà», dirá Berlusconi montado sobre la puerta de su coche, en un discurso que ha pasado a la historia como la «rivoluzione del predellino» ${ }^{10}$. La elección de la plaza San

10 «Berlusconi show. Forza Italia addio, nuovo partito», Corriere della Sera, 19-11-2007. 
Babila no fue casual. En Milán el lugar está asociado a los encuentros y movilizaciones de jóvenes neofascistas de la galaxia política del Movimento Sociale Italiano (MSI) contra las organizaciones estudiantiles comunistas y de extrema izquierda, que en los años setenta se organizaba en el entorno urbano de la universidad de Milán. Se trataba, por tanto, de un desafío simbólico en toda regla a la clase dirigente de Alleanza Nazionale guiada por Fini.

El golpe de escena de Berlusconi estaba orquestado para reducir la autonomía y maniobrabilidad de UdC de Casini y AN de Fini frente a la opinión pública. Empero, Casini renunciaría a disolver su partido para confluir en el PdL con un sonoro "Caro Silvio, non siamo in vendita». Con ese gesto Casini ponía fin a catorce ańos de colaboración entre $\mathrm{FI} \mathrm{y} \mathrm{UdC}^{11}$. Gianfranco Fini, por su parte, también había acariciado la idea de liderar un proyecto para la derecha italiana, dando por amortizada la figura de Berlusconi tras la derrota en las elecciones de $2006^{12}$. Sin embargo, la debilidad de AN en las encuestas electorales, unido a la presión de algunos nombres de peso de la clase dirigente de su partido a favor del entendimiento con Berlusconi, hicieron que Fini aceptase disolver AN para dar vida a un nuevo partido junto a Berlusconi (Cicchito, 2019: 130-131).

\section{CARATERÍSTICAS IDEOLÓGICAS DEL PDL}

El nacimiento del PdL tuvo su reflejo en una oferta ideológica diferenciada para el electorado de la derecha italiana. Como advertía Angelo Panebianco en un editorial del Corriere della Sera, "Quien piense que el Popolo della Libertà es solo una continuación de Forza Italia y Alleanza Nazionale quizás se equivoca». Para el politólogo italiano el nuevo partido de Berlusconi llevaba una impronta en el plano simbólico-identitario que lo diferenciaba sustancialmente de Forza Italia. Si este había sido desde 1994 el partido de la «revolución liberal», el PdL nacía como el partido de la «reforma del Estado». La idea de la reforma de las instituciones, subrayaba Panebianco, formaba parte de los programas de Forza Italia ab initio, pero estaba subordinada a la realización de la «revolución liberal» que debía liberar a la sociedad civil del Estado. Sin embargo, el PdL, un partido que nacía con una grave crisis económica en ciernes, armonizaba su propuesta ideológica con un "prepotente retorno del Estado» ${ }^{13}$.

11 «Casini rompe col PdL», la Repubblica, 16-02-2008.

12 «Fini: Centrodestra può stare senza Silvio», la Repubblica, 16-12-2007.

13 A. Panebianco, «La lunga partita a tre», Corriere della Sera, 30-03-2007. 
Ciertamente, con la puesta de largo del PdL la derecha liderada por Berlusconi concretaba una transición ideológica hacia un registro conservador, tanto en lo económico y social como en lo identitario. Un discurso que dejaba atrás el optimismo por el progreso universal del liberalismo que Forza Italia encarnó en los años noventa en sintonía con la tesis del «fin de la Historia» de Francis Fukuyama y en consonancia con el contexto posterior a la caída del comunismo. Un nuevo discurso diferenciado, también, del ideario democristiano que había ocupado el programa del partido a partir de la incorporación de Forza Italia al Partido Popular Europeo en 1998. Una redefinición ideológica que traducía, en última instancia, la voluntad de dar forma a un nuevo bloque de poder de signo netamente conservador a partir de la fusión con el mundo postfascista de Alleanza Nazionale, el diálogo abierto con el electorado de la Lega Nord y el alejamiento definitivo del popolarismo de la UdC.

\section{LA CONSTRUCCIÓN DE UN PARTIDO CONSERVADOR}

El estilo de liderazgo político desplegado por Silvio Berlusconi a lo largo de la Segunda República ha sido identificado, no sin acierto, como un elemento clave para explicar el proceso de personalización y desideologización de la política italiana. Sobre todo para la constitución de un modelo de «partido personal» en el que el líder y sus necesidades estratégicas, por encima de tradiciones y culturas políticas, se convierten en la medida de la política (Calise, 2010; Bordignon, 2014). Ciertamente, tanto en el caso de Forza Italia como en el del Popolo della Libertà, el hiperliderazgo ejercido por Berlusconi ha sido diagnosticado como uno de los factores que han comprometido la institucionalización organizativa y la coherencia programática de sus partidos (Raniolo, 2006: 443).

Sin embargo, siguiendo la estela de Panebianco, la impronta de Berlusconi sobre la evolución del liderazgo y los modelos de partido en Italia debe ser compatible con otorgar al análisis ideológico un espacio diferenciado. Sobre todo porque el estudio de la progresiva redefinición en el tiempo de su oferta programática ofrece información relevante sobre la transformación del territorio de caza sobre el que Berlusconi y sus cuadros han aspirado a ejercer influencia y dominio, de modo que la naturaleza programática de los partidos liderados por Berlusconi no puede ser interpretada sin atender tanto a su estrategia para con el resto de partidos de la derecha italiana como a la fuerza organizadora del sistema de competición bipolar que preside la Segunda República. En este sentido, una de las características ideológicas fundacionales del PdL que le diferenciaba de Forza Italia fue la desaparición de toda referencia a elementos de izquierda liberal y reformista como fuente de identidad 
del partido. Como diría Berlusconi en el discurso de apertura del Primer Congreso Nacional del PdL, el partido nacía con la aspiración de ser «un gran partido mayoritario de los moderados, de todos los italianos que no se reconocen en la izquierda» (Berlusconi, 2013: 145).

En la Carta dei valori que FI aprobó en su Segundo Congreso, celebrado en 2004, el partido se definía como «un sujeto inédito para la historia de Italia", señalando que "no se adapta, por tanto a Forza Italia, la definición de centro-derecha. Somos más bien un partido de centro, liberal-popular y liberal-socialista, aliado de la derecha moderada, abierto a la cultura de la izquierda reformista» (Forza Italia, 2004: 25). Esta vocación de apertura a la izquierda reformista, a la cultura liberal y al mundo católico por parte de FI, expresaba una vocación permanente en el partido desde su fundación en 1994: representar el espacio electoral ocupado por el pentapartito - la coalición de Gobierno compuesta por el DS, PSI, PSDI, PRI y PLI como oposición al PCI- entre 1981 y 1992, más allá de la coherencia ideológica. En este sentido, la Carta dei valori de 2004, en el apartado titulado "Las fuentes de nuestra identidad», señalaba como origen y fundamento de la identidad del partido personalidades vinculadas al mundo que representaba la galaxia política del pentapartito:

Nos movemos sobre todo en la gran área del humanismo cristiano y laico, el espacio que ha inspirado las tradiciones político-culturales más importantes de la historia del país. En la trayectoria del pensamiento y la obra de De Gasperi y Einaudi, en la inspiración federal y liberal de Cattaneo y Sturzo, en el universo laico y socialista que lleva de Salvemini hasta Calamandrei, Maranini, Ugo La Malfa, Malagodi, Saragat, Craxi (Forza Italia, 2004: 21).

Esta apertura ideológica también sirvió a Forza Italia para absorber a una buena parte de la clase política que tras el proceso judicial Tangentopoli se había quedado sin un partido de referencia. Resulta cierto que Silvio Berlusconi había cultivado de manera importante la imagen de Forza Italia como un partido nacido ex novo, fundado por un outsider de la política y que aspiraba a portar una nueva clase política las instituciones del Estado (Ignazi, 2008: 60). Todo ello al objeto de sintonizar con el clima antipolítico que se generó en Italia tras la quiebra del sistema político en el periodo 1992-1994. Como dirá el mismo Berlusconi en el famoso discurso televisado en el que anunció su decisión de presentarse a las elecciones de 1994, «la vieja clase política italiana ha sido barrida por los hechos y superada por los tiempos» (Berlusconi, 2013: 3). Sin embargo, como ha puesto de manifiesto el profesor Ilvo Diamanti, en los grupos parlamentarios de FI la presencia de políticos que provenían de los partidos de la Primera República desaparecidos en el periodo 1992-1994 fue 
relevante y estuvo guiada por una dinámica creciente. En las elecciones de 1996 el $38 \%$ de los políticos de Forza Italia provenía del algún partido del pentapartito, mientras que tras las elecciones de 2001 el porcentaje de cargos con origen en la Primera República aumenta hasta el 46\%, con especial presencia de políticos provenientes de la DC y el PSI (Diamanti, 2009: 135).

Sin embargo, la estructura ideológica que se hace presente en la Carta dei valori del PdL, aprobada en el Primer Congreso del partido celebrado en 2009, muestra una ruptura clara con la vocación de representar al mundo de la izquierda liberal y reformista que había caracterizado el proyecto político de Forza Italia desde su puesta en liza ${ }^{14}$. Más allá de las apelaciones retóricas a todos los italianos por encima de divisorias políticas, en la Carta dei valori del PdL se impone una distinción binaria entre derecha e izquierda a modo de visiones antagónicas del mundo:

La izquierda siempre ha traído a Italia incertidumbre, división, odio social y pobreza. La izquierda hace políticas que destruyen la familia y que no respetan los valores morales del pueblo italiano, los valores de nuestra tradición. Por lo que siempre ha sido en su sentido profundo, la izquierda quiere dividir a los trabajadores de los empresarios, a los hombres de las mujeres, a los padres de los hijos, a los jóvenes de los ancianos, a los italianos del norte de los italianos del sur. La izquierda ve enemigos por doquier. Nosotros vemos donde sea a nuestros iguales (Popolo della Libertà, 2009).

El análisis de la evolución programática de los partidos liderados por Berlusconi no es arbitraria, sino que responde a una lógica o racionalidad interna. A modo de ejemplo, a medida que a partir de 1996 se asienta la naturaleza bipolar del sistema político italiano, Forza Italia irá absorbiendo el ala derecha de la diáspora democristiana, huérfana de representación política tras la desaparición de la DC y la alianza del mundo socialcristiano con el universo excomunista. El reflejo programático de esta democristianización de Forza Italia, que culmina con la integración del partido en el PPE, se hace evidente en el abandono de su discurso liberal original, marcado por el modelo

14 Véase, por ejemplo, el elogio de la figura de Bettino Craxi como «socialista autonomista, reformista, humano y anticomunista» en el acto de homenaje al líder del PSI celebrado en Roma, en el Palacio San Macuto, en 2002 (Berlusconi, 2006: 123-130). En el discurso de apertura del Primer Congreso Nacional del PdL, Berlusconi también homenajeará a Craxi como el primer presidente en dirigirse al MSI «superando así la idea de que la verdadera Constitución italiana era el acuerdo entre la Democracia Cristiana y el Partido Comunista» (Berlusconi: 2013: 146). 
anglosajón de Reagan y Thatcher, para incorporar a su visión de la política la cultura política democristiana representada por Luigi Sturzo y Alcide de Gasperi ${ }^{15}$.

La transición de Forza Italia hacia el registro ideológico democristiano se aprecia de manera considerable, por ejemplo, en la adopción de la economía social de mercado: «Mercado y equidad social no son conceptos antagónicos como nos han querido hacer creer cierto catolicismo social y la ortodoxia de la izquierda. Son, al contrario, conceptos gemelos [...]. Nuestro liberalismo no es por tanto salvaje, sino social» (Forza Italia, 2004: 17). En el subrayado de la dimensión religiosa del liberalismo: «No es casualidad que la primacía asignada al ser humano y su libertad constituye un motivo de íntima y profunda vecindad, cuando no identidad, entre el pensamiento cristiano y el pensamiento liberal» (ibid.: 27-28). O en la concepción del europeísmo como proyecto político: «Nosotros somos europeístas de siempre, pero no somos eurofanáticos. En nuestro cromosoma, por expresarme con el lenguaje utilizado por un alto exponente de nuestra mayoría, está el verdadero europeísmo, el de Alcide de Gasperi, de Konrad Adenauer y de Robert Schuman, no el de los conversos de última hora» (Berlusconi, 2001: 254).

Frente al desarrollo ideológico que presenta la evolución programática de Forza Italia desde 1994 a 2004, la Carta dei valori del PdL aprobada en 2009, expresión programática de la fusión de FI y AN, adquiere una naturaleza revolucionaria. Sobre todo porque desaparece la vocación de representar el espacio político del pentapartito, y con ello cualquier referencia a las tradiciones liberales y reformistas, laicas y católicas, que habían operado como las fuentes de su identidad. En concreto, de la Carta del PDL desaparece el liberalismo como concepto nuclear, así como todas las referencias al mundo democristiano - De Gasperi, Sturzo, Schuman y Adenauer - a través de las cuales Forza Italia legitimaba y articulaba su visión de la libertad, la sociedad, el mercado y la visión de Occidente como miembro del PPE.

La nueva Carta del PdL propone a los italianos «una sociedad hecha de libertad y desarrollo económico, de solidaridad». Donde se propone «una sociedad basada en valores liberales y cristianos, sobre la familia natural fundada en el matrimonio, formada a partir de la unión de un hombre y una mujer, en la que hacer nacer, crecer y educar a los hijos» (Popolo della Libertà, 2009). La alusión a "valores liberales» no esconde que la centralidad que el «liberalismo» había tenido en Forza Italia, se desvanece. En su lugar se abre

15 Véase, por ejemplo, el discurso de Berlusconi sobre Sturzo del 23 de noviembre de 2005, con ocasión de la inauguración de una placa en su honor en la sede de Forza Italia (Berlusconi, 2006: 139-142). 
paso una visión acentuadamente identitaria y conservadora que refleja con claridad la separación de la cultura política del popolarismo y la fusión con el universo ideológico de Alleanza Nazionale, que lleva a Berlusconi a las antípodas del discurso quasi libertario con el que se estrenó en 1994.

Con el liderazgo de Gianfranco Fini, Alleanza Nazionale, heredero del MSI, se había liberado del peso de la tradición neofascista que había mantenido al partido prácticamente aislado durante la Primera República. Sin embargo, Fini aprovechó el contexto de oportunidad creado por el hundimiento del sistema de partidos de posguerra en el periodo 1992-1994 para transformar el partido y liderar una mutación ideológica orientada a crear una organización abierta a un votante conservador, caracterizado por una visión comunitaria, social y marcadamente espiritual de la política (Tarchi, 2003; Ignazi, 2004).

La naturaleza de este nuevo discurso identitario se hace evidente en el tratamiento de las ideas de Europa y Occidente. En la Carta de Forza Italia de 2004 se asigna «un gran valor a la idea de Occidente, como expresión de la civilización liberal y cristiana» que culmina la honda expansiva de la «revolución americana como evento clave de la filosofía pública liberal que se contrapone a la revolución jacobina de París» (Forza Italia, 2004: 29). Mientras que la Carta del PdL se apoya en una visión «espiritual», vinculada a «las raíces judeo-cristianas de Europa y la herencia común cultural clásica y humanista, con la mejor parte de la Ilustración" que delinea, de fondo, una idea de Europa más cercana a la visión "gaullista» de la Europa de los pueblos que aquella concepción federalista de Europa propia del universo democristiano al que se adhiere Forza Italia en 1998 (Popolo della Libertà, 2009).

También se hace presente a la luz del tratamiento del fenómeno de la inmigración. En la Carta de Forza Italia de 2004 se conjugaba el deber de acogida de inspiración cristiana «de aquel que busca nuestra tierra un futuro mejor» con el deber de respetar «nuestra cultura, nuestra religión, nuestra tradición, nuestras leyes» (Forza Italia, 2004: 36). Sin embargo, en la Carta del PdL el problema de la inmigración se inscribe en el territorio diferenciado de la seguridad: "Nosotros pensamos que se debe sumar a la libertad otro valor, que la complementa: la seguridad de nuestra identidad frente a la inmigración» (Popolo della Libertà, 2009). Al señalar la inmigración no como un problema de integración, sino como un peligro existencial para la identidad de Occidente, Berlusconi llevó el PdL al encuentro con la posición que tanto Alleanza Nazionale como la Lega compartían frente a la inmigración musulmana o de origen islámico.

En este punto, los tres partidos de la derecha italiana - FI, LN, ANmostraban sintonía con las distintas interpretaciones de la tesis del «choque de civilizaciones» del politólogo norteamericano Samuel P. Huntington, la cual 
se había abierto paso como referencia en el mundo intelectual conservador a partir de los atentados del 11 de septiembre de 2001, invitando a fortalecer la identidad occidental ante un mundo abierto al conflicto cultural y decantando, en el caso de AN y LN, un cambio radical de ambos partidos en relación con su posición frente a EE. UU., cuya cultura política capitalista, individualista y universalista habían criticado en el pasado desde postulados nacionalistas, si bien diferenciados y enfrentados (Tarchi, 2003; Ignazi, 2004). «En un mundo desafiado por el terrorismo y atravesado por el riesgo del choque de civilizaciones, nosotros proponemos la construcción de la paz y el diálogo entre pueblos como deber fundamental de nuestra política internacional» (Popolo della Libertà, 2009) ${ }^{16}$.

En todo caso, si el anuncio de la fundación del PdL como casa común de la derecha en Italia fue un golpe de escena orquestado por Berlusconi para neutralizar la autonomía del resto de partidos de la derecha, la transición de Forza Italia hacía un registro ideológico más conservador y menos liberal no responde a la improvisación. Al contrario, se trata de una estrategia política, madurada a la luz del modelo del neoconservadurismo americano, que aspiraba a elaborar una cultura política católica postdemocristiana, en diálogo abierto con la Iglesia y movimientos católicos, como cemento ideológico para la construcción de un partido de vocación mayoritaria con el que enfrentarse al PD en el marco de competición bipolar.

Este nuevo conservadurismo, tal y como fue delineado por intelectuales de peso en la esfera de Forza Italia como Marcello Pera, Gaetano Quagliariello, Baget Bozzo o Giuliano Ferrara, conocidos como «teocon» en la opinión pública, pretendía recuperar la religión como principio inspirador y organizador del orden político ${ }^{17}$. Articulándolo ad extra como americanismo y

16 La Lega Nord dirigida por Umberto Bossi se opuso a la intervención de la OTAN en Kosovo en 1999 en consonancia con la posición original del partido contra el imperialismo de los EE. UU. Su tradicional postura antislámica se traducirá en un giro proamericano en política exterior a partir de los atentados del 11S (Ignazi, 2004: 155). En el caso de Alleanza Nazionale, la disolución del MSI acompaña la eliminación de los residuos de un antiamericanismo de signo nacionalista a favor de un reconocimiento del papel de los EE. UU. en el mantenimiento del orden y la libertad en el mundo a través de la OTAN y a un reconocimiento expreso del Estado de Israel (Tarchi, 2003: 165-166).

17 Véanse, por ejemplo, las publicaciones de estos intelectuales y políticos de Forza Italia sobre el tema en la editorial Mondadori, propiedad de Berlusconi: G. Quagliariello, Cattolici, pacifisti, teocon. Chiesa e politica in Italia dopo la caduta del Muro (Mondadori, 2006); M. Pera, Perché dobbiamo dirci cristiani. Il liberalismo, l'Europa, l'etica (Mondadori, 2008); M. Pera y J. Ratzinger, Senza radici: Europa, relativismo, 
antislamismo y proyectándolo ad intra como una posición de resistencia contra el relativismo moral y la deriva laicista de la izquierda, que encuentra su cénit en el caso Eluana Englaro (Colarizi y Gervasoni, 2012: 134-136). ${ }^{18}$

En el discurso de apertura de Silvio Berlusconi en el Primer Congreso Nacional del PdL aún se registra la huella del proyecto de la «revolución liberal», con menciones a los procesos revolucionarios del XviII, que operó en Forza Italia como marca de la casa desde su fundación, sobre todo como diagnóstico que calificaba Italia como una «democracia incompleta» a merced, a lo largo del siglo $\mathrm{xx}$, de pulsiones totalitarias a izquierda y derecha (Berlusconi, 2013: 145). Sin embargo, si en el programa de Forza Italia el equilibrio entre catolicismo y liberalismo se decantaba a favor del segundo, en el programa del PdL la relación se invierte. Este cambio reflejaba el progresivo acercamiento de la derecha berlusconiana a la Iglesia desde el final del papado de Juan Pablo II y la Conferencia Episcopal Italiana, presidida por el cardenal Camillo Ruini. Un acercamiento y colaboración que se consolidaría con el papado de Benedicto XVI, que hace de la derecha berlusconiana el interlocutor privilegiado de la Iglesia ${ }^{19}$.

Nótese, sin embargo, que esta operación conservadora orientada a hegemonizar la representación de una determinada forma de entender la cultura católica en la esfera política italiana no solo buscaba dar un nuevo impulso al anticomunismo dos décadas después de la caída del muro, presentando un nuevo escenario bipolar en base a la fractura religiosa del mundo. Perseguía, también, a partir del debate y movilización pública sobre cuestiones morales y religiosas —el aborto, la eutanasia, el matrimonio gay, etc.- complicar la convivencia en el seno del PD de dos tradiciones políticas diferenciadas, la exdemocristiana y la excomunista, que no habían sido capaces de dar forma a una nueva cultura política compartida ${ }^{20}$. De fondo, por tanto, se diseñaba una estrategia de movilización social y protesta en torno a cuestiones morales

cristianesimo, Islam (Mondadori, 2004); G. Baget Bozzo, Lintreccio. Cattolici e comunisti 1945-2004 (Mondadori, 2004), y G. Ferrara, Non dubitare. Contro la religione laicista (Solfanelli, 2005).

18 "Quagliariello: Non è morta è stata ammazzata», Corriere della Sera, 09-02-2009.

19 Para medir la importancia de la fractura religiosa en la política italiana, como la convergencia de la derecha berlusconiana con las posiciones de la Iglesia, véase el debate en torno a la aprobación de la Ley 40/2004 sobre Reproducción Asistida y el referéndum abrogativo celebrado en 2005 (Martini, 2006: 164-181).

20 Para entender la relevancia del entendimiento entre la DC y el PCI y sus consecuencias para el mundo católico en el imaginario político de FI y el PdL, véase la elaboración histórico-teórica de G. Baget Bozzo L'Intreccio. Cattolici e comunisti 1945-2004 (Mondadori, 2004). 
que buscaba restaurar la unidad del mundo católico italiano en torno a un solo partido de la derecha. Las declaraciones de Berlusconi en el llamado Family Day del 2007, movilización popular en defensa de la familia tradicional, marcan el tono de la batalla cultural desplegada por el entorno de Forza Italia para señalar la contradicción entre la izquierda y el catolicismo: «No a las familias de serie B, no a quien quiere reducir la Iglesia a una Iglesia en silencio como en la Unión Soviética, no a los católicos que están en la izquierda: se contradicen ${ }^{21}$.

\section{LA CONTINUIDAD DEL DISCURSO ANTICOMUNISTA}

Buena parte del éxito de Silvio Berlusconi se ha basado en su capacidad para entender que el consenso anticomunista que determinó la política de la Primera República, encarnada en la conventio ad excludendum frente al PCI, seguía operativo como fuerza movilizadora a partir de 1994. En las diferentes etapas programáticas que pueden advertirse en la trayectoria de los partidos de Berlusconi, desde Forza Italia al Popolo della Libertà, el anticomunismo está presente como el mínimo denominador común que ha funcionado como el cemento ideológico que ha mantenido unido al centro-derecha en Italia por encima de las discrepancias internas.

El argumentario anticomunista puesto en circulación por Silvio Berlusconi y su entorno se apoya en la idea en virtud de la cual el colapso de la URSS no produjo en Italia el mismo resultado político que en el resto de países de Occidente: la desaparición de los partidos comunistas y la deslegitimación de su ideología. Al contrario, el diagnóstico que opera en el berlusconismo subraya que la coincidencia en el tiempo de la caída del muro de Berlín y el proceso judicial Mani pulite determinó un final de la Guerra fría paradójico para la política italiana. A saber, que los comunistas sobreviviesen al tiempo histórico de la Guerra Fría mientras que los partidos que habían movilizado el consenso anticomunista desaparecían tras el colapso del bloque soviético (Orsina, 2009: 79; Orsina, 2013: 119). En el simbólico «Discorso nel decennale della caduta del muro di Berlino» Berlusconi ofrece una de las expresiones más claras de este diagnóstico:

El PCI-PDS, el partido que debería haberse empeñado en una profunda y despiadada autocrítica denunciando su propia responsabilidad en el apoyo, ofrecido por más de setenta años, a la dictadura totalitaria del comunismo soviético — exhibido como el «paraíso de los trabajadores» antes que como el

21 «Berlusconi contro i cattolici di sinistra», la Repubblica, 12-05-2007. 
«archipiélago Gulag»— se encontró, de golpe, promocionado al rango de campeón de la democracia y único partido con las «manos limpias», mientras que las fuerzas democráticas se encontraron sentados de golpe en el banco de los imputados, acusados de ser la caja de Pandora de la corrupción. El muro de Berlín había caído, pero no sobre la cabeza de los vencidos, sino sobre la cabeza de los vencedores (Berlusconi, 2013: 69).

Como ha señalado el politólogo Marco Tarchi, Berlusconi ha cultivado al electorado tradicionalmente hostil a la izquierda, huérfana de representación política tras la desaparición de los partidos que encarnaron la oposición al PCI bajo la forma del pentapartito en el periodo 1992-1994, presentándose como el "uomo della provvidenza» llamado a evitar el acceso de los herederos del comunismo al Gobierno de Italia (Tarchi, 2015: 283). Esta idea puede apreciarse con fuerza en el famoso discurso televisivo de enero de 1994, conocido como el discurso de la "discesa in campo", en el que Berlusconi anunció su decisión de concurrir a las elecciones que se celebrarían en abril de ese mismo año:

Nuestras izquierdas dicen haber cambiado. Dicen haberse convertido en liberal-democráticas. Pero no es verdad. Sus hombres son siempre los mismos, su mentalidad, su cultura, sus convicciones más profundas, sus comportamientos parece iguales. No creen en el mercado, no creen en la iniciativa privada, no creen en el beneficio, no creen en el individuo. No creen que el mundo pueda mejorar a través de la libre aportación de personas distintas. No han cambiado (Berlusconi, 2013: 4-5).

A pesar de la profunda transformación programática e ideológica que se registra desde los primeros pasos de Forza Italia hasta la fundación del Popolo della Libertà, el diagnóstico anticomunista, que incide en la continuidad ideológica y organizativa del PCI y sus herederos, permanece como la identidad central del berlusconismo. Subraya, por tanto, la falsa conversión del comunismo italiano a los principios de la democracia liberal a pesar de la efectiva disolución del PCI. Tanto es así que el mismo diagnóstico se mantiene, con mayor rotundidad si cabe a pesar de la distancia histórica con 1989, en el discurso de apertura del Primer Congreso Nacional del PdL, celebrado en Roma en marzo de 2009:

En 1994 el PCI hacía poco que se había transformado en PDS, manteniendo intacto, del partido comunista, la estructura, todo el grupo dirigente, el centralismo democrático, hasta la hoz y el martillo. Pero, sobre todo, no renegando nunca de las ideas condenadas para siempre por la historia — si bien el muro de 
Berlín había sido derribado en 1989- y considerando que para reinventarse bastaba con sustituir, simplemente, una palabra: «demócratas» por «comunistas». [...] En Italia los únicos que han sobrevivido al fin de las ideologías han sido los derrotados de la historia. Como consecuencia, no existe, y no ha existido jamás, discontinuidad de estrategias y personal político entre la clase dirigente que era la heredera de Palmito Togliatti y la presente (Berlusconi, 2013: 147-149).

La estrategia de movilización del electorado del centro-derecha italiano puesta en marcha por Berlusconi, consistente en proyectar sobre la vida política de la Segunda República la fractura ideológica de la Guerra Fría, permite ver hasta qué punto la tesis de la novedad radical del berlusconismo puede ser refutada. Sobre todo porque los partidos de Berlusconi han explotado a fondo la fractura comunismo/anticomunismo propia de la cultura política de la Primera República, estableciendo una fuerte identidad entre ambos periodos, a pesar de la radical mutación del sistema de partidos y la naturaleza de la competición política (Salvadori, 2013: 175-176). En este mismo sentido, tomando como referencia el trabajo clásico Mappe dell'Italia política del politólogo Ilvo Diamanti puede observarse que las coaliciones electorales guiadas por Berlusconi entre 1994 y 2008 reproducen geográficamente, en términos de rendimiento electoral, la fractura anticomunista que condicionó la vida política italiana de la Primera República, desde 1948 a 1994. A mayor abundamiento, los territorios donde las coaliciones lideradas por Berlusconi se han mostrado tradicionalmente competitivas desde los años noventa, son las mismas zonas donde el Frente Popular, formado por el PCI y el PSI, mostró mayor debilidad electoral en 1948. Hasta tal punto, siguiendo a Diamanti, de poder afirmar que en la política italiana la fractura ideológica introducida en la Guerra Fría con el muro de Berlín fue sustituida en la Italia posterior a 1989 por el efecto del «muro de Arcore», en referencia a la villa milanesa propiedad de Silvio Berlusconi (Diamanti, 2009: 212-214).

El diagnóstico de Berlusconi sobre la continuidad del comunismo del PCI fuera del tiempo histórico de la Guerra Fría ha servido, al menos, a dos objetivos. En primer lugar, señalar a Italia como un país anómalo en el orbe político occidental debido a que «los derrotados de la historia» han sobrevivido al descrédito del comunismo en Europa. En segundo lugar, y como consecuencia de la primera tesis, justificar su competición con la izquierda italiana como algo más que una lucha por el poder y la posibilidad de ocupar el Estado: como una empresa cuyo objetivo es normalizar, democratizar y modernizar el país para armonizarlo con Occidente, donde los valores de la democracia liberal son hegemónicos.

La idea que asocia la competición política de Berlusconi a la lucha contra un experimento político fallido cuyo modelo amenaza con arrastrar a Italia fuera del 
mundo occidental se expresa con claridad en el discurso televisado de 1994: «He decidido saltar al campo y ocuparme de la cosa pública porque no quiero vivir en un país iliberal, gobernado por fuerzas inmaduras y hombres ligados doblemente a un pasado política y económicamente fallido» (Berlusconi, 2013:3). Y atraviesa toda la historia de Forza Italia, como muestra la coincidencia simbólica del Primer Congreso Nacional de Forza Italia de 1998 con la fecha del 18 de abril, en homenaje a la mayoría absoluta obtenida por De Gasperi en 1948: «Nosotros queremos firmemente, sin vacilaciones ni dudas, cultivar, ver crecer, cuidar y ver florecer el bien más precioso y delicado que nos han dejado en herencia los grandes italianos que el 18 de abril de hace cincuenta ańos eligieron Occidente y rechazaron las lisonjas del comunismo totalitario» (Berlusconi, 2001: 276).

La principal novedad que caracteriza al anticomunismo de Berlusconi en la etapa del Popolo della libertà es la voluntad de profundizar la fractura comunismo/anticomunismo proyectándola sobre la ruptura americanismo/antiamericanismo que toma cuerpo en el mundo tras los ataques del $11 \mathrm{~S}$ con el corolario de la invasión de Irak en 2003. La línea propuesta por Giuliano Ferrara a partir del 11S, diputado de Forza Italia, periodista y fundador de Il Foglio, sintetiza la dirección neoconservadora que va a tomar el partido hasta su consolidación en la Carta dei valori del PdL. El trabajo periodístico de Ferrara será crucial para reformular el paradigma clásico que asociaba comunismo y antiamericanismo en la Guerra fría y proyectarlo al mundo post-11S como un nuevo paradigma que traducía el antiamericanismo de la izquierda italiana como una posición proislámica, en un país donde Berlusconi aparecía como el nuevo campeón del americanismo y el atlantismo (Colarizi y Gervasoni, 2012: 134). De este modo, la posición prooccidental que había tratado de capitalizar el anticomunismo clásico de Forza Italia irá más allá de la defensa clásica del liberalismo, en su vertiente política y económica, para pasar a elaborar un discurso identitario sobre la naturaleza de Occidente, orientado, en última instancia, a integrar el bloque de la derecha italiana a la luz de los nuevos conflictos culturales de un mundo atravesado por el «choque de civilizaciones», utilizando la expresión del politólogo norteamericano Samuel P. Huntington.

En este sentido, la fundación del Popolo della libertà, como proyecto conservador, culmina en su programa la progresiva elaboración de un discurso por parte del mundo intelectual de Forza Italia donde la izquierda heredera del PCI es señalada como la aliada del islam por su defensa del laicismo y el multiculturalismo. Como señalará el polémico sacerdote y asesor de Berlusconi, Gianni Baget Bozzo, «un multiculturalismo que tiende exactamente a disgregar el carácter católico de la tradición nacional $\aleph^{22}$. En la misma línea, en

22 G. Baget Bozzo «Quei cattolici di facciata dell'Unione», Il Giornale, 17-02-2006. 
2006, en el encuentro anual que Comunión y Liberación celebra en Rimini, Berlusconi aprovechó para afirmar que «ila izquierda pretende un país multiétnico y multicultural y nosotros pensamos al contrario, nosotros queremos la Italia católica de los italianos! $\aleph^{23}$.

\section{CRISIS Y DISOLUCIÓN DEL POPOLO DELLA LIBERTÀ}

El Popolo della Libertà, el gran proyecto de Silvio Berlusconi para dar una casa común a la derecha italiana, fue disuelto el 16 de noviembre de 2013. La crisis y disolución del PdL no puede ser disociada de tres cuestiones fundamentales que ponen fin a la trayectoria política de Berlusconi en la XIV Legislatura. En primer lugar, la dimisión de Berlusconi como presidente del Consejo de Ministros de Italia el 16 de noviembre de 2011 y la formación del Gobierno tecnocrático de Mario Monti, un hecho que no puede desvincularse del impacto de la crisis económica mundial en el sur de Europa, que provocó la dimisión de José Luis Rodríguez Zapatero en España, Silvio Berlusconi en Italia y Yorgos Papandreu en Grecia en un periodo de tiempo excepcional de dieciocho días (Bosco y Verney, 2012). En segundo lugar, la condena en firme a cuatro ańos de prisión e inhabilitación para ostentar cargo público impuesta a Berlusconi por fraude fiscal en el caso Mediaset, confirmada el 1 de agosto de 2013 por el Tribunal Supremo. Condena que provocó, a la postre, su expulsión del Senado el 27 de noviembre del mismo año ${ }^{24}$. Y en tercer lugar, la difícil convivencia de los liderazgos de Berlusconi y Fini — presidente de la Cámara de los Diputados entre 2008 y 2013 - en el seno del PdL ${ }^{25}$.

No obstante, el proceso de disolución del PdL y su fracaso como partido conservador tampoco puede desvincularse de los efectos sobre el comportamiento del electorado que se derivan de la profunda transformación del sistema de partidos que se produce en Italia en el periodo 2011-2013. Una mutación que conduce al fin del bipolarismo que había caracterizado la competición política de la Segunda República. Esta dinámica bipolar, como se ha mostrado, no solo había racionalizado la competición política en

23 «Berlusconi da Cl: l'Italia sia cattolica e degli italiani», Corriere della Sera, 26-08-2006.

24 "Berlusconi condannato: ma resto in campo", Corriere della Sera, 02-08-2013; «Berlusconi non è più senatore. Senato approva la decadenza», la Repubblica, 27-112013.

25 El 29 de julio de 2010 Gianfranco Fini abandonó el PdL junto a 34 diputados y 10 senadores para formar un nuevo grupo parlamentario, núcleo del futuro partido Futuro e libertà per l'Italia (Cichitto, 2019: 160-162). 
términos organizativos; a saber, incentivando la creación de dos grandes partidos, el PD y el PdL, como instrumento para transitar del bipolarismo al bipartidismo. El bipolarismo también había contribuido a la reorganización, si bien con problemas y dificultades, de la oferta ideológica de la izquierda y la derecha en Italia, profundizando en la identidad progresista y conservadora de los dos grandes partidos. Para el caso de Berlusconi, la quiebra del bipolarismo va a suponer el fin de la competición entre izquierda y derecha en la que el magnate milanés había encontrado su espacio natural proyectando progresivas interpretaciones del conflicto de la Guerra Fría fuera de su tiempo histórico.

\section{EL FIN DEL SISTEMA DE COMPETICIÓN BIPOLAR DE LA SEGUNDA REPÚBLICA}

En las elecciones de 2008 las coaliciones lideradas por el PD y el PdL consiguieron el mayor consenso jamás logrado por partidos prosistema en Italia, el 84,36\%. Sin embargo, en las elecciones celebradas en 2018 ese capital se vio drásticamente reducido al 41,6\%. En lo que toca a Berlusconi, si en las elecciones de 2008 el PdL consiguió una victoria reseńable al obtener un consenso del $37,38 \%$, el mejor resultado logrado por un partido liderado por el Cavaliere desde 1994, en las elecciones de 2018, concurriendo de nuevo con la refundada Forza Italia, su apoyo disminuyó bruscamente hasta el $14,00 \%$, perdiendo, por primera vez en la historia del berlusconismo, la condición de partido hegemónico de la derecha en Italia a favor de la lepenizada Lega de Matteo Salvini (Valbruzzi, 2018: 158-160) ${ }^{26}$.

Esta drástica pérdida de peso electoral del PdL y el PD en las elecciones de 2018, los mismos partidos que en 2008 habían llevado a su máximo rendimiento el bipolarismo, se produce de forma progresiva. Nótese que en las cruciales elecciones de 2013, marcadas por la irrupción y victoria electoral del partido populista Movimento Cinque Stelle (M5S), fundado por el cómico Beppe Grillo, el consenso conjunto de las coaliciones lideradas por el PD y el PdL bajó al 58,68 \%. En el caso del PD, descendió del 33,18\% al 25,43\% de los votos. En el caso del PdL, bajó del 37,38\% al 21,56\%.

La espectacular incorporación del M5S al sistema de partidos provocó la evolución del mismo hacia una nueva forma de competición tripolar. Un cambio radical en la correlación de fuerzas de los partidos italianos en liza, que para algunos autores justifica cuestionarse la vigencia misma de la llamada Segunda República, dado que su criterio distintivo, como fue señalado

26 Los datos electorales utilizados provienen del Archivo Histórico del Ministerio del Interior de Italia. Disponible en: https://elezionistorico.interno.gov.it/. 
anteriormente, había sido el bipolarismo (D’Alimonte, 2013: 128). Sin embargo, antes de que la irrupción del M5S llevase a término oficial la ruptura con la era de la competición bipolar en las elecciones de 2013, esta se había producido de facto con un pasaje crucial que determinará la política italiana de la década 2008-2018: la formación del Gobierno tecnocrático de Mario Monti en noviembre de 2011, con el apoyo parlamentario decisivo del PD, PdL y UDC (Pasquino y Valbruzzi, 2015: 445). Dando forma a una suerte de Gobierno de gran coalición, basada en el entendimiento entre el PD y el PdL (y sus sucedáneos), que marcará, con distintos grados e intensidades en la colaboración, el signo político de la XVII Legislatura (2013-2018) al extenderse como fórmula anti-M5S en los Gobiernos de Enrico Letta, Matteo Renzi y Paolo Gentiloni. Una nuevo tipo de conventio ad excludendum contra el populismo que después también se extenderá a la Lega, los dos partidos más votados en las elecciones de $2018^{27}$.

Desde una dimensión ideológica, la transición al sistema tripolar de competición, mediada por la irrupción del M5S, introduce una cesura notable en el registro discursivo de la Segunda República. El discurso populista del M5S se abre paso en un contexto de crisis económica y política, circunstancia que permite al movimiento de Beppe Grillo imprimir un registro moralista al debate público, apoyándose en un diagnóstico de la política donde la principal fractura de las sociedades contemporáneas se produce entre el pueblo y la élite, donde el primero es bueno y virtuoso por naturaleza, frente a la condición corrupta de los segundos. Este diagnóstico populista ha permitido al M5S apelar a un electorado transversal, presentándose como un partido postideológico que opera al margen de las divisiones ideológicas tradicionales, neutralizando así las diferencias entre el PD y el PdL al igualarlos en la categoría de «élite», "casta», «oligarquía» o «establishment» (Tarchi, 2015; Bordignon y Ceccarini, 2013).

La sustitución del eje izquierda-derecha por el eje arriba-abajo que realiza el M5S ha tenido como resultado práctico la construcción de un electorado transversal que se ha distribuido entre un grupo del $40 \%$ que rechaza las etiquetas izquierda y derecha, un 10\% que se considera de centro y dos grupos del 20-25\% que se consideran electores de centro-izquierda y centro-derecha. La capacidad del M5S para dar expresión al electorado de la derecha italiana quedó demostrada con sus victorias en las elecciones municipales de 2016 en Roma y Turín frente a candidatos del PD, por delante de los candidatos tradicionales de la derecha (Diamanti, 2014: 8; Ceccarini y Bordignon, 2016: 132).

27 Para una descripción detallada de la colaboración PdL (después Forza Italia)-PD a partir de 2011, incluido el Pacto del Nazareno, véase Martín de Santa Olalla (2018). 
Las elecciones de 2018 no hicieron sino confirmar la capacidad del populismo para crecer a costa de los dos principales partidos del sistema italiano, PD y PdL/FI. Los dos partidos más votados, el M5S con el 32,68\% de los votos y la Lega con el $17,35 \%$, se han caracterizado por su capacidad para romper con la lógica del voto en clave izquierda-derecha típica de la Segunda República. La Lega penetrando en el electorado clásico de la izquierda en la llamada "zona rossa» y el M5S captando el voto del PdL/FI en el centro y sur de Italia. El éxito de ambos partidos expresa una posición antipolítica de desconfianza frente al establishment político y una demanda de protección en un contexto de crisis política y económica. Protección cultural en el caso de la Lega, protección social en el caso del M5S (Valbruzzi y Vignati, 2018: 8-9).

\section{LA METAMORFOSIS DE LA DERECHA ITALIANA}

La disolución del PdL en noviembre de 2013 no solo puso fin al intento de crear un bloque de poder conservador en torno a un único partido dentro de la estructura de competición bipolar de la Segunda República. La crisis y desaparición del PdL se tradujo en la metamorfosis de la derecha italiana a partir de su división en cuatro nuevos partidos: Forza Italia, refundada en noviembre de 2013; Nuovo Centro Destra, escisión del PdL liderada por el otrora delfín de Berlusconi, Angelino Alfano; Fratelli d'Italia, escisión del PdL liderada por Giorgia Meloni, y la nueva Lega de Matteo Salvini. Mientras que los dos primeros iban a competir por representar al electorado moderado del centro-derecha, Fratelli d'Italia y la Lega jugarían la carta de la nueva derecha soberanista, con especial especial éxito, en esta transformación, para la lepenizada Lega de Salvini (Tarchi, 2018).

No deja de ser paradójico observar que la explosión del populismo en Italia, que convertirá en el periodo 2013-2018 al M5S y la Lega en los partidos que más crecen en número de votos, haya desdibujado la posición política de Berlusconi. Principalmente porque el propio discurso de Berlusconi ha sido clasificado como populista. Como han puesto de manifiesto los principales intérpretes del populismo italiano, en el discurso de Berlusconi se hace presente un registro antipolítico que se encarna en un elogio permanente de la forma movimiento frente a la forma partido, de la sociedad civil frente al Estado, del sentido común frente a la ideología y del ciudadano frente al político profesional (Orsina, 2013; Tarchi, 2015). Como se ha tratado de mostrar con el análisis de la dinámica de oposición al Gobierno Prodi en el periodo 2006-08, que lleva a la fundación del PdL, también comparable con la oposición realizada en 1996-1998, el registro populista de Berlusconi emerge con fuerza como estrategia de crítica y deslegitimación del Gobierno, explotando la conexión entre el líder carismático y el pueblo frente al poder (Del Palacio, 2017: 244). 
El análisis de la génesis y desarrollo del discurso populista de Berlusconi muestra, sin embargo, hasta qué punto su éxito ha dependido de una estructura bipolar en el que se desplegaba la lógica de oposición a la izquierda, identificada con la pervivencia del comunismo en el mundo post-1989. Dicho de otro modo, el populismo de Berlusconi, a diferencia del desplegado por el M5S o la Lega, ha buscado reforzar la fractura izquierda-derecha trabajando sobre el eje comunismo-anticomunismo. Nótese que el juego de oposiciones movimiento-partido, sociedad-Estado, sentido común-ideología, ciudadano-político, adquieren sentido en la lógica del anticomunismo (Orsina, 2013:118-119). Incluso en su registro conservador, al que se ha aludido en la sección dedicada a la elaboración ideológica del PdL, la progresiva apelación al carácter católico del pueblo italiano aparece como un bien objetivo que proteger de la pulsión secularizadora del comunismo y sus herederos, al punto de equiparar la misión de Forza Italia y Berlusconi en Italia con la de los movimientos democráticos de Europa del Este, cuya lucha contra el comunismo expresaba, también, la voluntad de recuperación histórica de la «democracia, nación, patria y soberanía» (Berlusconi, 2013: 177).

Esta fuerte dependencia del discurso de Berlusconi de un sistema político bipolar, en el cual poder movilizar un anticomunismo de corte conservador y comunitarista como elemento agregador de la derecha, ayuda a explicar, junto a otros factores, el carácter ambiguo y vacilante de la política del Cavaliere tras las elecciones de 2013, cuya expresión última es el resultado electoral de 2018, el peor registro en la trayectoria política de Berlusconi. Si en 2011, como presidente del Consejo de Ministro aún podía apelar a su misión como baluarte frente al comunismo italiano, tras la política de colaboración con el PD para sostener el Gobierno de Mario Monti y la firma del llamado Pacto del Nazareno en enero de 2014 con Matteo Renzi, líder con una clara vocación centrista, la deslegitimación del partido heredero del PCI fue perdiendo razón de $\operatorname{ser}^{28}$. De otra parte, dado el flujo de voto desde el PdL y FI hacia el M5S, en clave de voto antipolítico, la estrategia de asociar al M5S con un nuevo tipo de comunismo, más peligroso que el original, no ha funcionado como recurso para reagrupar el voto de la derecha en torno a Berlusconi ${ }^{29}$.

28 El llamado Pacto del Nazareno recogía un acuerdo entre los líderes del PD y FI, Renzi y Berlusconi, para promover una reforma de la Constitución, la conversión del Senado en una cámara regional, y la aprobación de una nueva ley electoral mayoritaria. El paco fue roto por Berlusconi tras la elección de Sergio Mattarella, a propuesta de Renzi, como presidente de la República (Tarchi, 2018: 156-157); «Berlusconi: Ancora vivo il pericolo comunista», Corriere della Sera, 26-02-2011.

«Berlusconi: M5S più pericolosi dei comunisti nel 94», La Stampa, 26-11-2017. 
Del mismo modo, la ambigüedad mostrada por Berlusconi frente al proyecto soberanista encarnado por la nueva Lega de Salvini tampoco ha contribuido a clarificar la posición política de Berlusconi. La identificación con algunas medidas de corte euroescéptico, como la crítica al euro, han puesto a Berlusconi en ruta de colisión con del Partido Popular Europeo, al que pertenece Forza Italia desde 1998. En definitiva, el apoyo mostrado a Salvini como nuevo líder de la derecha ha terminado por legitimar la liquidación del centro-derecha, como espacio autónomo y diferenciado, para dejar su espacio a un populismo que reivindica la política como enfrentamiento pueblo versus élite, amén de una marcada eurofobia, dando por clausurada la etapa de la política italiana donde la lucha por el poder se dirimía entre izquierda y derecha ${ }^{30}$.

\section{CONCLUSIONES}

La radical transformación de la derecha italiana que tiene lugar en la década 2008-2018 no puede entenderse sin analizar al fracaso del Popolo della Libertà y sus consecuencias. Como se ha tratado de mostrar, el PdL nace de la fusión de Forza Italia y el partido postfascista Alleanza Nazionale con la intención de reunir al electorado de la derecha italiana en torno a un proyecto de signo conservador, nacido para aprovechar a fondo la dinámica bipolar de la competición política de la Segunda República. Sin embargo, la interrupción del IV Gobierno Berlusconi, formado tras las elecciones de 2008, resultado de la dimisión del Cavaliere en noviembre de 2011, inicia la crisis que termina con el recorrido PdL en 2013 y da lugar a la metamorfosis de la derecha italiana, dejando, en definitiva, sin continuidad el proyecto de construcción de un partido conservador que se inicia en torno a 2004 y que culminó la fundación del PdL.

En este artículo se ha tratado de mostrar que la fundación del PdL no puede entenderse sin atender a dos factores. Primero, la evolución del sistema de partidos de la Segunda República, cuya naturaleza bipolar incentivó la racionalización organizativa e ideológica del sistema en torno a dos grandes partidos. Segundo, la dinámica de oposición al segundo Gobierno de Romano Prodi (2006-2008), marcado por la progresiva identificación del partido con una agenda conservadora en torno a cuestiones de signo ético, religioso e identitario.

30 «Berlusconi: Con la doppia moneta si rilanciano i consumi», Il Giornale, 20-08-2017; «Salvini liquida il centrodestra: la prospettiva è popolo contro élite», Corriere della Sera, 22-05-2018. 
A partir de esta premisa, este artículo ha tratado de mostrar que la pérdida de consenso de los partidos liderados por Silvio Berlusconi en la década 20082018 también se puede explicar en clave ideológica, en función de la irrupción de un nuevo modelo de competición tripolar en medio de la crisis económica que supera el eje ideológico izquierda-derecha, poniendo a prueba la capacidad de adaptación de los viejos partidos del sistema al nuevo entorno político. Un modelo, el que se afirma en Italia a partir de las elecciones de 2013, que supera la confrontación izquierda-derecha, reduciendo de manera drástica la tradicional capacidad de Berlusconi para movilizar, agrupar e integrar al electorado hostil a la izquierda apelando a una lectura conservadora del anticomunismo italiano como pretendía el PdL.

\section{BIBLIOGRAFÍA}

Bartolini, S., Chiaramonte, A. y D'Alimonte, R. (2004). The Italian Party system between Parties and Coalitions. West European Politics, 27 (1), 1-19. Disponible en: https://doi. org/10.1080/01402380412331280783.

Berlusconi, S. (2001). Discorsi per la democrazia. Milano: Mondadori.

- (2006). Verso il partito della libertà. Lidentita, i valori, il progetto. Milano: Mondadori.

- (2013). Discorsi per la libertà. Milano: Mondadori.

Biorcio, R. (2015). Il populismo nella politica italiana. Da Bossi a Berlusconi. Da Grillo a Renzi. Milano: Mimesis.

Bordignon, F. (2014). Il partito del capo. Da Berlusconi a Renzi. Rímini: Maggioli

- y Ceccarini, L. (2013). Five Stars and a Cricket. Beppe Grillo Shakes Italian Politics. South European Society and Politics, 18 (4), 427-449. Disponible en: https://doi.org/10. 1080/13608746.2013.775720.

Bosco, A. y Verney, S. (2012). Electoral epidemic: The political cost of economic crisis in Southern Europe. South European Society and Politics, 17 (2), 129-154. Disponible en: https://doi.org/10.1080/13608746.2012.747272.

Calise, M. (2010). Il partito personal. I due corpi del leader. Roma: Laterza.

Ceccarini, L. y Bordignon, F. (2016). The Five Stars continue to shine: the consolidation of Grillo's movement party in Italy. Contemporary Italian Politics, 8 (2), 131-159. Disponible en: https://doi.org/10.1080/23248823.2016.1202667.

Cicchitto, F. (2019). Storia di Forza Italia, 1994-2018. Soveria Mannelli: Rubbettino.

Colarizi, S. y Gervasoni, M. (2012) La tela di Penelope. Storia della Seconda Repubblica. Roma: Laterza.

Cota M. y Verzichelli, L. (2007). Political Institutions in Italy. Oxford: Oxford University Press.

D'Alimonte, R. (2013). The Italian elections of February 2013: The end of the Second Republic?. Contemporary Italian Politics, 5 (2), 113-129. Disponible en: https://doi.org/ 10.1080/23248823.2013.807599. 
Del Palacio, J. (2017). El populismo en Italia: el berlusconismo y su legado. En A. Rivero, J. Zarzalejos y J. del Palacio (coords.). Geografía del populismo. Un viaje por el universo del populismo desde sus orígenes hasta Trump (pp. 234-247). Madrid: Tecnos.

Diamanti, I. (2009). Mappe dall'Italia politica.Bianco, verde, rosso, azzurro... e tricolore. Bologna: Il Mulino.

- (2014). The 5 Stars Movement: a political laboratory. Contemporary Italian Politics, 11 (1), 43-62. Disponible en: https://doi.org/10.1080/23248823.2014.881016.

— y Lello, E. (2005). The Casa della Libertà: A House of Cards? Modern Italy, 10 (1), 9-35. Disponible en: https://doi.org/10.1080/13532940500113326.

Forza Italia (2004). Carta dei valori. Disponible en: http://download.repubblica.it/pdf/forzaitalia_valori1.pdf.

Grilli di Cortona, P. (2007). Il cambiamento politico in Italia. Dall Prima alla Seconda Repubblica. Roma: Carocci Editore.

Ignazi, P. (2004). Changing guard in the Italian extreme right. Representation, 40 (2), 146-156. Disponible en: https://doi.org/10.1080/00344890408523256.

- (2008). Partiti politici in Italia. Bologna: Il Mulino.

Martín de Santa Olalla, P. (2018). Italia, 2013-2018. Del caos a la esperanza. Madrid: Liber Factory.

Martini, Ch. (2006). The Referendum on Artificial Insemination. En G. Amyot y L. Verzichelli (eds.). Italian politics. The End of Berlusconi era? (pp. 163-181). Oxford: Berghahn Books. Disponible en: https://doi.org/10.3167/ip.2005.210110.

Moroni, C. (2008). Da Forza Italia al Popolo della Libertà. Roma: Carocci.

Orsina, G. (2009). The Republic after Berlusconi: Some reflections on historiography, politics and the political use of history in post 1994 Italy. Modern Italy, 15 (1), 77-92. Disponible en: https://doi.org/10.1080/13532940903488713. (2013). Il berlusconismo nella storia d'Italia. Venezia: Marsilio.

Panebianco, A. (2009). Modelos de partido. Organización y poder en los partidos políticos. Madrid: Alianza Editorial.

Pasquino, G. y Valbruzzi, M. (2015). The impact of the 2013 general elections on the italian political system: The end of bipolarism? Journal of Modern Italian Studies, 20 (4), 438-453. Disponible en: https://doi.org/10.1080/1354571X.2015.1066111.

Popolo della libertà. (2009). Carta dei valori. Disponible en: http://www.pdl.it/notizie/15346/ carta-dei-valori.

Raniolo, F. (2006). Forza Italia: A Leader with a Party. South European Society and Politics, 11 (3-4), 439-455. Disponible en: https://doi.org/10.1080/13608740600856470.

Salvadori, M. (2013). Storia d'Italia. Crisi di regieme e crisi di sistema, 1861-2013. Bologna: Il Mulino.

Tarchi, M. (2003). The political culture of Alleanza Nazionale: an analysis of the party's programmatic documents (1995-2002). Journal of Modern Italian Studies, 8 (2), 135-181. Disponible en: https://doi.org/10.1080/1354571032000078248.

- (2015). Italia populista. Dal Qualunquismo a Beppe Grillo. Bologna: Il Mulino.

- (2018). Voters without a party. The «Long Decade» of the Italian Centre-Right and its Uncertain Future. South European Society and Politics, 23 (1), 147-162. Disponible en: https://doi.org/10.1080/13608746.2018.1434454. 
Valbruzzi, M. (2018). Analisi elettorale di un cambiamento radicale. Chi a vinto e chi a perso. En M. Valbruzzi y R. Vignati (eds.). Il vicolo cieco. Le elezioni del 4 marzo 2018 (pp. 147-184). Bologna: Il Mulino; Istituto Carlo Cattaneo.

— y Vignati, R. (eds.) (2018). Il vicolo cieco. Le elezioni del 4 marzo 2018. Bologna: Il Mulino; Istituto Carlo Cattaneo. 